\title{
O DOADOR MARGINAL: EXPERIÊNCIA DE UM CENTRO DE TRANSPLANTE DE FÍGADO
}

\section{The marginal donor: a single-center experience in orthotopic liver transplantation}

\author{
Olival Cirilo Lucena da FONSECA-NETO, Luiz Eduardo Correia MIRANDA, Bernardo David SABAT, \\ Américo Gusmão AMORIM, Luiz ADEODATO, Paulo Sérgio Vieira de MELO, Helry Cândido LOPES, \\ Cláudio Moura LACERDA, Leila Maria Moreira Beltrão PEREIRA
}

ABCDDV/580

Fonseca-neto OCL, Miranda LEC, Sabat BD, Amorim AG, Adeodato L, Melo PSV, Lopes HC, Lacerda CM, Pereida LMMB. O doador marginal: experiência de um centro de transplante de fígado. ABCD Arq Bras Cir Dig 2008;21(1):1-5

RESUMO - Racional - Desde que o uso de enxertos marginais é solução aceita para escassez de órgãos para transplante, ele tornou-se muito comum em todo mundo e a literatura vem mostrando efetividade desses enxertos no transplante de fígado. Objetivo - Apresentar a experiência do Serviço de Transplante Hepático do Hospital Universitário Oswaldo Cruz, em transplante de fígado com o uso de doadores marginais. Métodos - Estudo retrospectivo em 137 transplantes ortotópicos de fígado, usando enxertos marginais entre 1999 e 2006, com acompanhamento mínimo de 180 dias. Os receptores foram classificados de acordo com a função inicial do enxerto no pós-operatório como normal (FN) e disfunção primária (DP). Resultados - Não foi observada diferença estatisticamente significante entre os grupos FN e DP com os seguintes parâmetros dos doadores: idade, sódio sérico, tempo de protrombina, esteatose hepática, transaminases sérica, pressão sanguínea, drogas vasoativas, índice de massa corpórea, parada cardíaca antes da doação de órgão, doador em assistolia e tempo de isquemia quente. Análise da curva de sobrevida (Kaplan-Meier) de pacientes e de enxertos de fígado de pacientes que receberam fígado de doadores ideais versus doadores marginais não mostrou diferença com significância estatística. Conclusão - Pode ser recomendado o uso de enxertos marginais para transplantes hepáticos, inclusive os provenientes de doadores com o coração parado.

DESCRITORES - Transplante de fígado. Doador marginal. Doador de órgãos. Cirrose hepática. Imunossupressão.

\section{INTRODUÇÃO}

Lesão por isquemia e reperfusão é processo inevitável que culmina com morte celular e afeta muito a função do enxerto após o transplante de fígado ${ }^{1}$. Em seguida à restauração do fluxo sangüíneo, o fígado é submetido a lesão adicional mais grave do que a induzida pela isquemia ${ }^{2}$. Disfunção primária e a sua forma mais grave, a falência primária, são conseqüências da lesão de isquemia e reperfusão e representam importante causa no aumento da morbidade e mortalidade após transplante de fígado ${ }^{3}$.

$\mathrm{O}$ uso de enxertos marginais tem sido solução aceita pela escassez de órgãos para transplante e o seu uso tornou-se muito comum em todo mundo. A literatura vem mostrando efetividade desses órgãos e seus resultados no transplante de fígado ${ }^{4,5,6}$.

O objetivo deste estudo é apresentar a experiência com transplante de fígado utilizando órgãos de doadores marginais em um centro hospitalar.

Trabalho realizado no Serviço de Cirurgia Geral e Transplante Hepático do Hospital Universitário Oswaldo Cruz - HUOC - UPE, Recife, PE, Brasil.

Endereço para correspondência: Olival Cirilo Lucena Fonseca Neto - E-mail: olivalneto@globo.com

\section{MÉTODOS}

Este é um estudo retrospectivo de todos os pacientes submetidos a transplante de fígado entre 1999 e 2006 no Serviço de Cirurgia Geral e Transplante Hepático do Hospital Universitário Oswaldo Cruz em Recife, PE, Brasil. Entre 178 transplantes consecutivos, 137 usaram enxertos marginais e participaram do estudo. Todos os pacientes foram informados sobre os procedimentos que envolviam o procedimento. O desenho e o protocolo de pesquisa foram aprovados pelo Conselho de Ética do hospital.

O procedimento de captação foi realizado após a definição de morte encefálica caracterizada segundo as leis brasileiras ${ }^{6,7}$. Os enxertos hepáticos foram retirados por técnica clássica com ressecção de veia cava, e resfriados com solução de Belzer. Os vasos ilíacos direito e esquerdo, foram retirados em todos os doadores no término do procedimento de captação. Os enxertos hepáticos foram implantados segundo técnica convencional sem bypass venovenoso, ou pela técnica de piggyback ${ }^{9}$. Os elementos do pedículo foram anastomosados e a reconstrução biliar foi realizada com colédoco-coledocostomia ou com colédoco-jejunostomia em Y de Roux. Biópsia intra-operatória do fígado não foi realizada. A esteatose hepática foi avaliada subjetivamente, considerando a cor do fígado e as características de sua borda. O protocolo em relação à imunossupressão consistiu de tacrolimus, prednisona 
e micofenolato de mofetil para adultos e tacrolimus com prednisona para crianças.

Foram estudados o tempo de acompanhamento mínimo de seis meses, o escore MELD, sódio sérico acima de 155 $\mathrm{mEq} / \mathrm{L}$, a PAM, o INR, transaminases séricas, utilização de drogas vasopressoras, o tempo de UTI maior que quatro dias e o índice de massa corpórea.

$\mathrm{O}$ doador foi definido como marginal se existisse pelo menos um dos seguintes critérios: idade acima de 55 anos; esteatose macroscópica acima de $30 \%$; hipotensão com pressão arterial média $(\mathrm{PAM})<100 \mathrm{mmHg}$; necessidade de drogas vasopressoras (qualquer dose de noradrenalina ou utilização de dose $>10 \mu \mathrm{g} / \mathrm{kg} / \mathrm{min}$ de dopamina); hipernatremia $>155 \mathrm{mEq} / \mathrm{L}$; tempo de permanência em UTI $>4$ dias; tempo de isquemia fria $>12$ horas; tempo de isquemia quente $>40$ minutos; transaminases com valores altos: aspartato aminotransferase (AST) $>140 \mathrm{mg} / \mathrm{dL}$ e alanina aminotransferase (ALT) $>170 \mathrm{mg} / \mathrm{dL}$; índice de massa corporal $(\mathrm{IMC})>30$; passado de parada cárdio-pulmonar antes da doação ou assistolia no momento da doação. Na ausência desses critérios o doador era considerado ideal.

Até julho de 2006, os receptores de transplante de fígado foram selecionados por critério cronológico ${ }^{16}$. Os pacientes foram classificados de acordo com a função hepática no pós-operatório como: função normal, pacientes com transaminases séricas abaixo de $2000 \mathrm{mg} / \mathrm{dL}$, tempo de protrombina (Razão Normalizada Internacional - INR) < 2, ou bilirrubina total $<10 \mathrm{mg} / \mathrm{dL}$ até o sétimo dia de pósoperatório. Pacientes que mostraram disfunção primária foram divididos em: a) com mau função primária, os que apresentavam transaminases séricas $>2000 \mathrm{mg} / \mathrm{dL}$, INR $>2$ ou bilirrubina total $>10 \mathrm{mg} / \mathrm{dL}$ até o sétimo dia de pós-operatório; b) com falência primária, pacientes que faleceram ou foram retransplantados durante os primeiros sete dias por não-funcionamento do enxerto.

Os resultados foram expressos através das médias e desvios-padrão. Os valores foram comparados através da análise univariada usando o teste de Mann-Whitney U - para variáveis contínuas e o teste exato de Fisher para variáveis categóricas. A análise de sobrevida foi realizada usando o método de Kaplan-Meier, com teste de log-rank para comparação das curvas. A curva de sobrevida dos pacientes que receberam enxertos ideais foi comparada com aqueles com enxertos marginais. A diferença assumida para significância foi $\mathrm{P}<0,05$. Análise estatística foi realizada usando o software Prism 4.0 (GraphPad software, San Diego, Califórnia, USA).

\section{RESULTADOS}

Entre 178 doadores de fígado estudados, 137 (76,97\%) foram classificados como marginais. Entre estes, existiam 73 homens $(53,3 \%)$ e 64 mulheres $(46,7 \%)$ com a média de idade de 33,3 $\pm 17,2$ anos ( 3 a 63 ). A causa do óbito foi hemorragia cerebral em 59 casos (43\%), trauma crânioencefálico em $52(38 \%)$, neoplasia do sistema nervoso central em $12(8,7 \%)$ e outras causas em 14 casos $(10,2 \%)$. Em 16 doadores $(11,7 \%)$ a idade estava acima de 55 anos.
Quanto aos dias de internamento na UTI, 35 pacientes $(25,5 \%)$ a permanência foi de quatro dias, com média de $3,8 \pm 2,8$ dias. A parada cardíaca antes da doação ocorreu em 26 pacientes $(19 \%)$, e em seis doadores $(4,4 \%)$ ocorreu captação com coração parado. A utilização de drogas vasopressoras ocorreu em 82 doadores (59,8\%). Em 24 $(17,5 \%)$ o nível das transaminases séricas era suficiente para classificá-los como doador marginal. O nível de INR estava acima de 2 em 21 doadores $(15,9 \%)$ e o sódio sérico acima de $155 \mathrm{mEq} / \mathrm{L}$ em 57 casos (41,6\%). Esteatose macroscópica foi encontrada e considerada acima de 30\% em 62 doadores $(45,25 \%)$ e o índice de massa corpórea acima de 30 foi observado em sete $(5,3 \%)$.

O tempo cirúrgico no grupo de função normal em relação à isquemia fria e quente foi respectivamente 442,8 minutos $\pm 169,5$ minutos $(n=102)$ e 59,9 minutos $\pm 43,2$ minutos ( $\mathrm{n}=102)$ Em $92,5 \%$ das vezes o tempo de isquemia quente foi acima de 40 minutos.

No grupo de mau-funcionamento primário o tempo de isquemia fria e quente foi respectivamente 447,5 \pm 167 minutos e $54,4 \pm 10,2$ minutos ( $\mathrm{n}=180$ ). Em $85,7 \%$ o tempo de isquemia quente foi acima de 40 minutos.

No grupo de falência primária o tempo de isquemia fria e quente foi respectivamente $377 \pm 143,7$ minutos e $47,5 \pm 15,2$ minutos $(n=50)$. Em $60 \%$ ocorreu tempo de isquemia quente acima de 40 minutos. Não houve diferença estatisticamente significante entre os grupos.

\section{Evolução pós-transplante e curvas de sobrevida}

Todos os pacientes tiveram tempo mínimo de acompanhamento mínimo de seis meses $(25,7$ a 301 semanas). Entre os receptores existiam 89 homens $(59,1 \%)$ e 56 mulheres $(40,9 \%)$ com a média de idade de $40,6 \pm$ 19,8 anos (6 meses a 71 anos). Quatorze mortes $(10,4 \%)$ ocorreram até o sétimo dia de pós-operatório. Apenas sete $(5,1 \%)$ foram devido à falência primária.

Entre os 137 pacientes que receberam enxertos hepáticos marginais, $112(81,7 \%)$ evoluíram com bom funcionamento no pós-operatório. Deste grupo, 60 eram homens $(53,6 \%)$ e 52 eram mulheres (46,4\%); o escore MELD foi $16,4 \pm 6,5, \mathrm{n}=86$. Oito doadores $(6,5 \%)$ tinham idade acima de 55 anos. Em 45 (40,1\%) o sódio sérico estava acima de $155 \mathrm{mEq} / \mathrm{L}$. Em 17 casos (15,1\%) o INR era superior a 2. Vinte e um doadores (18,7\%) apresentavam transaminases séricas altas. A utilização de drogas vasopressoras ocorreu em 65 doadores (58\%). Em 26, o tempo de UTI foi maior que quatro dias $(23,2 \%, 3,72 \pm 2,9$ dias). Ocorreram seis casos $(5,3 \%)$ de doadores com o coração parado e todos apresentaram boa evolução clínica e em 21 (18,7\%) houve parada cardíaca antes da doação. A PAM foi de $80,82 \pm$ 10,79 em 99 doadores e em sete $(6,2 \%)$ o índice de massa corpórea estava acima de 30.

Entre os pacientes que apresentaram mau-funcionamento primário $(\mathrm{n}=18,13,1 \%)$ o escore MELD era 21,8 $\pm 11(\mathrm{n}=13)$. Três doadores $(16,6 \%)$ apresentavam idade acima de 55 anos. Em oito $(44,4 \%)$ o doador apresentava sódio sérico acima de $155 \mathrm{mEq} / \mathrm{L}$. Em 10 doadores (55,5\%) 
existia a utilização de drogas vasopressoras. Em sete $(38,9 \%)$ o tempo de permanência em UTI foi maior que quatro dias; apenas em um ocorreu parada cardíaca antes da doação; o nível de INR estava acima de 2 em dois pacientes. O nível de transaminases estava elevado em 11,1\%. A PAM dos doadores foi $80,6 \pm 10,72 \mathrm{mmHg}(\mathrm{n}=16)$. Não foi encontrado doador com IMC acima de 30.

Entre os pacientes que evoluíram com falência primária $(\mathrm{n}=7,5,1 \%)$, um doador apresentava idade acima de 55 anos. Em cinco casos $(71,4 \%)$ o doador apresentava sódio sérico acima de $155 \mathrm{mEq} / \mathrm{L}$. Nos sete doadores foi encontrada utilização de drogas vasopressoras. Em quatro $(57,1 \%)$ o tempo de permanência de UTI foi acima de quatro dias; em três (43\%) a parada cardíaca ocorreu antes da doação. Em dois o INR estava acima de 2. O nível sérico de transaminases estava alto em apenas um doador e nenhum apresentava IMC acima de 30. A PAM foi de 80,5 $\pm 10,2 \mathrm{mmHg}(\mathrm{n}=5)$. Não foi possível calcular o MELD no momento do transplante.

Não houve diferença estatisticamente significante entre os grupos função normal e disfunção primária com as seguintes variáveis do doador: idade, sódio sérico, INR, esteatose hepática, transaminases séricas, PAM, utilização de drogas vasopressoras, IMC, parada cardíaca e doador com coração parado.

A análise das curvas de sobrevida (Kaplan-Meier) para pacientes que receberam fígado de doadores ideais versus doadores marginais não mostrou diferença estatisticamente significante $(P=0,67, \log$-rank test, Figura $1 \mathrm{~A})$. Não existiu diferença significante das curvas de sobrevida do entre o enxerto ideal e o marginal $(P=0,13, \log$-rank test, Figura 1B). A sobrevida dos pacientes após seis meses que receberam enxertos de doadores normal e marginal foi $82 \%$ e $72 \%$, respectivamente $(P=0,30)$. A sobrevida do enxerto após seis meses foi de $80 \%$ para aqueles provenientes de doadores ideais e de $71 \%$ para os marginais $(P=0,31)$. A sobrevida após um ano dos pacientes e dos enxertos para doador normal comparados com o marginal foi $79,5 \%$ e $67,9 \%$ (paciente, $P=0,23$ ); $80 \%$ e $68,8 \%$ (enxerto, $P=0,23$ ).
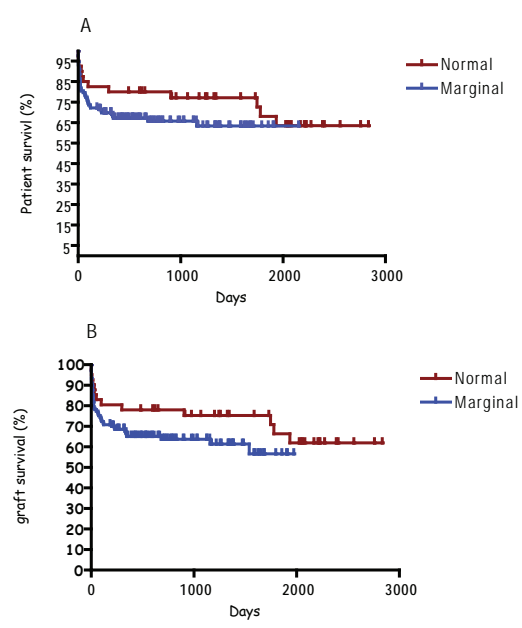

FIGURA 1 - Curvas de sobrevida para os receptores (A) e enxertos (B)
Análise das curvas de sobrevida dos pacientes, de seis meses e um ano, quando comparadas entre doadores ideais e marginais não mostraram diferença estatística $(P=0,9 \mathrm{e}$ 0,13 respectivamente, log-rank test, Figura 2). Não houve diferença significativa entre as curvas de sobrevida do enxerto de seis e 12 meses $(P=0,22$ e $P=0,13$ log-rank test, Figura 2).
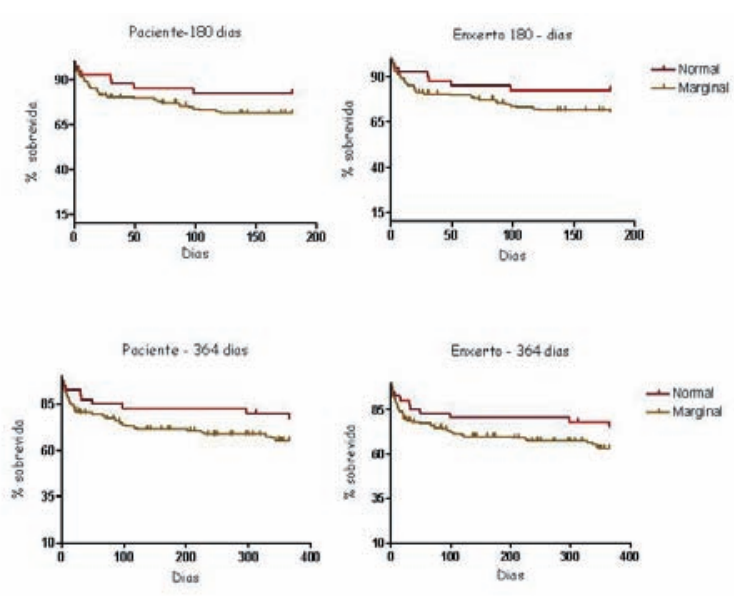

FIGURA 2 - Curvas de sobrevida (Kaplan-Meier) para receptores de transplante de fígado que receberam fígado de doador normal $(n=40)$ versus receptores de fígado de doador marginal $(\mathrm{n}=133)$ : a análise das curvas mediante o uso do log-rank teste não mostrou diferenças significantes entre os pacientes que receberam enxerto de doador marginal ou doador normal ao final de 180 e 364 dias.

\section{DISCUSSÃo}

O acompanhamento no pós-operatório de 180 dias foi utilizado nesse estudo porque esse é o tempo em que as complicações mais freqüentemente ocorrem em transplante de fígado. A lesão de isquemia e reperfusão é responsável pela disfunção primária do enxerto e pela sua apresentação mais grave, a falência primária. A escassez de órgãos para transplante obrigou a utilização de enxertos classificados como sub-ótimos, nos quais existe grande suscetibilidade para a lesão de isquemia e reperfusão ${ }^{18,19,20}$. Entretanto persiste relação incerta entre a qualidade desses órgãos e a expectativa de vida nos receptores de transplante com órgãos marginais. Apesar da importância dessa alternativa, não existe consenso sobre a caracterização do doador marginal ${ }^{21}$.

Com a persistente escassez de órgãos muitos centros não realizam transplantes com enxertos com mais de 50\% de esteatose. Não existe concordância com o grau de esteatose do enxerto necessária para causar impacto nos resultados pós-transplante hepático. De fato, não existe consenso sobre o melhor método para investigar esteatose hepática nos enxertos ${ }^{22}$. Biópsia hepática realizada antes da retirada do órgão no doador parece ser a melhor opção para classificar e quantificar a esteatose, mas não é realizada rotineiramente. Avaliação ultrassonográfica do fígado do 
doador não permite diferenciar em macro ou micro esteatose. Nesta série de casos não houve diferença na esteatose macroscópica classificada subjetivamente pelo cirurgião da captação de órgãos com bases em achados anatômicos, entre os grupos função normal e disfunção primária. Classificação macroscópica da esteatose não serviu para decidir sobre a utilização dos órgãos nos doadores.

Idade do doador e hipernatremia está relacionada à alta mortalidade pós-operatória, mas novamente, os estudos publicados são conflitantes ${ }^{23}$. Não existe consenso sobre a idade do doador que comprometeria a função do enxerto. A maioria dos centros de transplante de fígado acreditam que fígados de doadores acima de 60 anos não deveriam ser utilizados para transplante. Entretanto, excelentes resultados obtidos com enxertos provenientes de doadores com idade acima de 80 anos já foram observados. Hipernatremia tem sido relacionada com má evolução no pós-operatório, mas essa sugestão não é confirmada por outros.

De modo similar, doadores com assistolia, parada cardíaca prévia a doação, alto nível de transaminases séricas, tempo de protrombina prolongado, tempo de intubação, PAM $<100 \mathrm{mmHg}$ e utilização de drogas vasopressoras são parâmetros utilizados com resultados controversos. Este trabalho teve seis doadores com coração parado. Em todos os casos a equipe cirúrgica estava presente quando diagnosticada a parada cardíaca e o fígado foi retirado prontamente. Todos os receptores que receberam fígado de doadores com coração parado tiveram excelente evolução pós-operatória. Esse resultado é semelhante aos centros de transplante de fígado de maior experiência ${ }^{24}$.

O tempo de isquemia fria prolongado ( $>12$ horas) pode influenciar negativamente na função do órgão no pós-operatório. No presente estudo, não existiu diferença estatística significante para o tempo de isquemia fria entre os grupos. No grupo de mau funcionamento do enxerto o maior tempo de isquemia fria foi de 7 horas e 27 minutos. O tempo de isquemia quente foi superior a 40 minutos na maioria dos pacientes e não houve diferença estatística para esse parâmetro entre os grupos. Com esses achados acredita-se que 40 minutos não seja o melhor cut-off no tempo de isquemia quente para definir órgão marginal.

Na presente série, não foi observada diferença estatística entre os grupos estudados para todas as variáveis consideradas. As curvas de sobrevida dos pacientes e enxertos não apresentaram diferenças estatísticas, assim como, as suas curvas de sobrevida de seis meses e um ano.

É importante ressaltarem-se alguns aspectos deste resultado. Primeiro, devido a dramática escassez de órgãos e a alta mortalidade na lista de espera, a maioria dos transplantes realizados no Serviço de Transplante Hepático do Hospital Universitário Oswaldo Cruz utilizou enxertos de doadores marginais ${ }^{25,26,27}$. Segundo, até junho de 2006, a alocação dos fígados de doadores cadáver obedecia ao critério cronológico. Por este fato, a maioria dos receptores até esta data apresentavam melhor reserva funcional (MELD mais baixo) e isso pode ter influenciado na boa evolução pós-operatória. Terceiro, o método de investigação da esteatose hepática escolhido não é o adequado e pode limitar as conclusões referentes a este importante parâmetro. Também é verdade que resultados referentes a transplante de fígado utilizando órgãos esteatóticos são controversos ${ }^{28}$. Quarto, a técnica de transplante hepático utilizada na maioria dos procedimentos foi a convencional sem desvio venovenoso no qual não foi observada influência negativa na evolução pós-operatória dos receptores que receberam enxertos marginais.

\section{CONCLUSÃO}

Pode ser recomendado o uso de enxertos marginais para transplantes hepáticos, inclusive os provenientes de doadores com o coração parado.

Fonseca-neto OCL, Miranda LEC, Sabat BD, Amorim AG, Adeodato L, Melo PSV, Lopes HC, Lacerda CM, Pereida LMMB. The marginal donor: a single-center experience in orthotopic liver transplantation. ABCD Arq Bras Cir Dig 2008;21(1):1-5

ABSTRACT - Background - Since marginal grafts are a solution to deal with the shortage of organ donors, its use became more common worldwide, and the literature had shown its effectiveness in the liver transplantation (LT) outcomes. Aim - To present a single center experience, at the Liver Transplantation Unit of Oswaldo Cruz University Hospital, with orthotopic LT using marginal organ donors. Methods - Retrospectivety review of 137 orthotopic LT using marginal grafts between 1999 and 2006, with a minimum 180 days follow-up. The receptors were classified according to postoperative initial graft function as normal function (NF) and primary dysfunction (PD). Results - No significant statistic difference was observed between groups NF and PD concerning the following donor's parameters: donor's age, serum sodium, prothrombine time, liver steatosis, serum transaminasis, blood pressure, vasoactive drugs, body mass index, heart attack prior organ donation, non-heart-beating donor and warm ischemia time. The survival curves' analysis (Kaplan-Meier) of patients or patients' grafts which received LT from ideal donors versus marginal donors showed no significant difference. Conclusions - The results permit to recommend the use of liver marginal grafts, including that ones from non-heart-beating donors.

HEADINGS - Liver transplantation. Directed tissue donation. Liver cirrhosis. Immunosuppression. 


\section{REFERÊNCIAS}

1. Busuttil RW. Emerging strategies to improve early graft function in ECDs. In: Satellite Symposium to the ILTS. Annual International Congress, 13. Expanding the Frontiers of Liver Transplantation 2007; 16-17.

2. De Wolfe A. Monitoring and handling of reperfusion. Liver Transpl Surg 1997; 3:459-461.

3. Brisceño J, Marchal T, Padillo J, Solórzano G, Pera C. Influence of marginal donors on liver preservation injury. Transplantation 2002; 74:522-526.

4. Mor E, Klintmalm GB, Gonwa TA, Solomon H, Holman MJ, Gibbs JF, Watemberg I, Goldstein RM, Husberg BS. The use of marginal donors for liver transplantation. A restrospective study of 365 liver donors. Transplantation 1992; 53:383-386.

5. Feng S, Goodrich NP, Bragg-Gresham JL, Dykstra DM, Punch JD, DebRoy MA, Greenstein SM, Merion RM. Characteristics Associated with Liver Graft Failure: The Concept of a Donor Risk Index. Am J Transplant 2006; 6:783790.

6. Conselho Federal de Medicina. Critérios para a caracterização da morte encefálica. Resolução $n^{\circ} 1480$ de 08/08/1997. Publicada no DOU de 21 de agosto de 1997; 18-227.

7. Decreto n 2268 - 30 de junho de 1997 - DOU 123, de 01/07/1997. Regulamenta a Lei n ${ }^{\circ} 9.434$, de 4 de fevereiro de 1997, que dispõe sobre a remoção de órgãos, tecidos e partes do corpo humano para fins de transplante e tratamento, e dá outras providências.

8. Avolio AW, Agnes S, Nure E, Gasbarrini A, Siciliano M, Pompili M, Castagneto $\mathrm{M}$. The Nonstandard liver, a hidden resource that cannot be overlooked: implications for the identification of the best recipient. Transplant Proc 2006; 38:1055-1058

9. Tzakis A, Todo S, Starzl TE. Orthotopic liver transplantation with preservation of the inferior vena cava. Ann Surg 1988; 20:649-652.

10. Busuttil RW, Farmer DG, Yersiz H, Hiatt JR, McDiarmid SV, Goldstein LI, Saab S, Steven HAN, Durazo F, Weaver M, Cao C, Chen T, Lipshutz GS, Holt C, Gordon S, Gornbein J, Amersi F, Ghobrial RM. Analysis of long-term outcomes of 3200 liver transplantations over two decades. Ann Surg 2005; 241:905-916.

11. Busuttil RW, Shaked A, Millis JM, Jurim O, Colquhoun SD, Shackletonnuesse BJ, Csete M Goldstein LG, McDiarmind SV. One thousand liver transplants, the lessons learned. Ann Surg 1994; 219:490-499.

12. Imber CJ, Shawn DSP, Handa A, Friend PJ. Hepatic steatosis and its relationship to transplantation. Liver Transplant 2002; 8(5):415-423.

13. Imber CJ, Shawn DSP, Lopez I, Guiver L, Friend P. Current practice regarding the use of fatty livers: a trans-atlantic survey. Liver Transplant 2002; 8(6):545549 .

14. Henderson JM. Liver Transplantation and rejection: an overview. HepatoGastroenterol 1999; 46(suppl 2):1428-1484.

15. ABT PL, Desai NM, Crawford MD, Forman LM, Markmann JW, Olthoff KM, et al. Survival following liver transplantation from non-heart-beating donors. Ann Surg 2004; 239:87-92.
16. Portaria $\mathrm{n}^{\circ} 1160$ - 29 de maio de 2006 - DOU 103 , de 31/05/2006. Modifica os critérios de distribuição de fígado de doadores cadáveres para transplante, implantando o critério de gravidade de estado clínico do paciente.

17. Klar E, Angelescu M, Zapletal C, Weiss G, Kraus TH, Herfarth CH. Predction of primary graft failure by intraoperative quantification of liver perfusion. Transplant Proc 2001; 33:1370-1371.

18. Renz JF, Kin CBA, Kinkhabwala M, Jan D, Varadarajan R, Goldstein M, Brown, R Jr., Emond JC. Utilization of extended donor criteria liver allografts maximizes donor use and patient access to liver transplantation. Ann Surg 2005; 242:556-563.

19. Busuttil RW, Tanaka K. The utility of marginal donors in liver transplantation. Liver Transpl 2003; 9:651-663.

20. Cameron AM, Ghobrial RM, Yersiz H, Farmer DG, Lipshutz GS, Gordon SA, Zimmerman M, Hong J, Collins TE, Gornbein J, Amersi F, Weaver M, Cao C, Chen T, Hiatt JR, Busuttil RW. Optimal utilization of donor grafts with extended criteria. A single-center experience in over 1000 liver transplants. Ann Surg 2006; 243:748-753.

21. Clavien PA. How far can we go with marginal donors? J Hepatol 2006; 45:483513.

22. López-Navidad A, Caballero F. Extended criteria for organ acceptance. Strategies for achieving organ safety and for increasing organ pool. Clin Transplant 2003; 17:308-324.

23. Totsuka E, Dodson F, Urakami A, Moras N, Ishii T, Lef MC, et al. Influence of high donor serum sodium levels on early postoperative graft function in human liver transplantation: effect of correction of donor hypernatremia. Liver Transpl Surg 1999; 5:421-428.

24. Tector AJ, Mangus RS, Chiestovich P, Vianna R, Fridell JA, Milgrom ML Sanders C, Kwo PY. Use of extended criteria livers decreases wait time for liver transplantation without adversely impacting posttransplant survival. Ann Surg 2006; 244:439-448.

25. Ferraz-Neto BH, Zurstrassen MP, Hidalgo R, Fonseca LE, Motta TD, Pandullo FL, Rezende MB, Meira-Filho SP, Sá JR, Afonso RC. Donor liver dysfunction: application of a new scoring system to identify the marginal donor. Transplant Proc 2007; 39(8):2516-8.

26. D'Albuquerque LA, Gonzalez AM, Filho HL, Copstein JL, Larrea FI, Mansero JM, Perón G Jr, Ribeiro MA Jr, Oliveira e Silva A. Liver transplantation from deceased donors serologically positive for Chagas disease. Am J Transplant 2007; 7(3):680-4

27. Rocha MB, Boin IFSF, Escanhoela CAF, Leonardi LS. Can the use of marginal liver donors change recipient survival? Transplant Proc 2004; 36:914-915.

28. Verran D, Kusyk T, Painter D, Fisher J, Koorey D, Strasser S, et al. Clinical experience gained from the use of 120 steatotic donor livers for orthotopic liver transplantation. Liver Transplant 2003; 9:500-505.

Conflito de interesse: não há Fonte financiadora: não há Recebido para publicação em: 23/06/2007 Aceito para publicação em: 04/10/2007 